\title{
An overview of the State Employed Special Interest Group (SESIG) of the South African Society of Psychiatrists (SASOP) from 2000 - 2012
}

\author{
Bernard Janse van Rensburg
}

SASOP SESIG National Convener (2008 - 2012) and Division of Psychiatry, University of the Witwatersrand, Johannesburg

Bernard Janse van Rensburg

Corresponding author: B Janse van Rensburg (bernardj@gpg.gov.za)

\begin{abstract}
Introduction. The State Employed Special Interest Group (SESIG) of the South African Society of Psychiatrists (SASOP) was established in Durban during the national congress in September 2000. Issues of concern at the time included: suboptimal physical conditions in state hospitals and clinics; stalling of the essential drug list (EDL) review process; and understaffing and difficulties in recruiting and retaining mental health medical personnel in the state sector. During the past 2 years, attention was given to liaising with the South African Medical Association (SAMA) as a medical labour organisation; standards for psychiatric inpatient structures, services and care; and scheduling a national SESIG strategic workshop.
\end{abstract}

Methods. A quantitative retrospective review of the demographic and occupational profile of SESIG's members, as captured by the SASOP database of current and potential members, was performed. The investigation included a review of the policies and processes by which strategic activities, priorities and measures for progress were identified within the different areas of SESIG's mandate.

Results. In 2007, 38\% (144) of the potential total number of stateemployed psychiatrists (380) were paid-up SESIG members; and 53\% (202) of the potential total number (378) in 2011. The Eastern Cape, Free State and Northern Gauteng subgroups had the biggest percentage of members per region in 2007, which changed in 2011 to Northern Gauteng, Western Cape and Eastern Cape. In 2011, $40 \%$ of the total membership comprised psychiatric registrars. Presentations and discussions at the first national strategic meeting of state-employed psychiatrists in 2012 covered: the scope of state sector practice; pertinent policies for state practice; planning per region; teaching and research; accepted principles for care; and strategic mobilisation (details in the supplement of this issue of the South African Journal of Psychiatry).

Conclusion. Eleven position statements were formulated to guide SASOP/SESIG activities during 2012 - 2014, relative to: national mental health policy; psychiatry and mental health; infrastructure and human resources; standard treatment guidelines and EDL; HIV in children and adults; substance abuse and addiction; community psychiatry and referral levels; recovery and re-integration; culture, mental health and psychiatry; the specialty status of South African psychiatry; and forensic psychiatry.

SAfr J Psych 2012;18(3):90-94. DOI:10.7196/SAJP.379

The State Employed Special Interest Group (SESIG) of the South African Society of Psychiatrists (SASOP) was established 12 years ago in Durban during the SASOP national congress in September 2000. Twenty-two members were present at this first meeting, with another 8 subsequent applicants. The first convener elected was Dave Swingler, and the interest group was duly constituted by January 2001. The first committee consisted of Yasmien Jeenah (Southern Gauteng), Margaret Nair (KwaZulu-Natal (KZN)), Sue Hawkridge (Western Cape), Renate du Preez (Northern Gauteng), Dave Swingler (Eastern Cape), Zygmunt Piotrowski (Free State) and a co-opted member from the Defence Force (Konrad Czech).

According to the SESIG constitution, the special interest group's purpose is to promote, maintain and protect the honour and interest of the discipline of psychiatry as a medical speciality in terms of the interests of medical practitioners and psychiatrists primarily in the employ of the state and who serve psychiatric patients within the public sector, as well as the interests of such patients and the community it serves. Ten objectives were identified, viz. to: foster good relationships among the members of the Group and the Society; promote co-operation with other associations involved in mental health; monitor, evaluate and advise policies related to the delivery of clinical services and the protection of patients' rights, especially within the public sector; promote research appropriate to psychiatry, especially within the public sector, in South Africa; promote appropriate training of undergraduate and postgraduate students in psychiatry; promote continuing education in psychiatry; 
maintain standards in psychiatry by peer review; promote and uphold the principles of human rights, dignity and ethics in the practice of public sector psychiatry; oppose unfair discrimination in the field of psychiatry; and promote the de-stigmatisation of psychiatry and increase the awareness of mental illness.

Main issues of concern identified as to be addressed by SESIG during the first 4 years of its existence included: suboptimal physical conditions of state hospitals and clinics; stalling of the essential drug list (EDL) review process; and understaffing and difficulties to recruit and retain mental health medical personnel in the state sector. These concerns were later categorised as: (i) patient-focussed issues (EDL, forensic services, hospital conditions, mental health legislation, disability grants, community care and liaison between the state, Lifecare and NGOs); and (ii) providerfocused issues (conditions of service, medical officers' status, double-load tensions - services delivery v. academic responsibility, service load, freezing of posts, foreign doctors and overtime issues). During 2002 and 2003, SESIG activities were in particular focused on the review of the EDL by the National EDL Committee. The SESIG convener and others (Dan Stein, Robin Emsley, Soraya Seedat and Solly Rataemane) were involved in the development of standard treatment guidelines (STG) to be integrated with the EDL. During 2004, the review of primary- and hospital-level EDLs continued. Completing his second 2-year term as convener, Dave Swingler recommended that: communication and contact with SASOP subgroups should be strengthened; communication across 'various domains' should be strengthened; focus should be placed on service delivery issues in the state sector; and efforts should be co-ordinated across provincial boundaries.

Christopher Szabo was elected to be the second convener at the national congress in September 2004 at the Champagne Sport Resort, Drakensberg. A new committee was formed, including Orlando Alonso-Betancourt (Eastern Cape), Bernard Janse van Rensburg (Southern Gauteng), Peter Milligan (Western Cape), Richard Nichol (Free State), Pierre Joubert (Northern Gauteng) and Shimina Sallojee (KZN), with registrar representatives Richard Sykes and Reyonold Marnewick. The EDL and conditions of service remained on the agenda, while other issues included: the proposed new Mental Health Care Bill; child and adolescent psychiatric beds; the 2-year medical internship; and accommodating registrars within SESIG. While the original 2-tiered approach regarding the rendering of service was still pursued during the subsequent 4 years (2005 - 2008), attention was given in particular to conditions of service related to remuneration packages and promotion within the state service, and on the compilation of a comprehensive list of psychiatrists and registrars in the country, in order to effectively canvass the SESIG constituency. During 2006, SESIG position statements on mental health facilities and staffing were drafted. These included 4 statements on: the responsibility of the state to provide mental healthcare infrastructure; current conditions of service in the public sector; and the management of care programmes, which were developed and circulated. These were presented and adopted at the national congress in Swaziland in September 2006.

Closer links with the South African Medical Association (SAMA) were also pursued. During 2007, Christopher Szabo was co-opted as a member of the national Tertiary and Quaternary EDL Expert Review Committee, and was also nominated to represent clinicians of the School of Clinical Medicine of the University of the Witwatersrand (WITS) on SAMA's Academic Doctors Association of South Africa (ADASA). During May 2007, an important submission to the South African Human Rights Commission was made by Thabo Rangaka, SASOP President at the time, on Obstacles on the Way for Patients to have Access to Health Care in South Africa. Major service delivery problems in the Eastern Cape became apparent during the second half of 2007, as communicated by Orlando Alonso-Betancourt at the time. This 4-year period was concluded with the notification of the Occupation Specific Dispensation (OSD) process in February 2008. Negotiations on the OSD for doctors was only completed in July of that year and therefore had to be implemented retrospectively. Specific liaison was pursued with SAMA at the time through SAMA's Public Sector (PUBSEC) Committee and ADASA, as was reported to the SASOP Executive in August 2008.

Bernard Janse van Rensburg was elected as convener, with Serame Maduna as deputy-convener at the national congress in September 2008 at Fancourt, George. The national committee for 2008 - 2010 included Peter Milligan (Western Cape), Mohammed Nagdee (Eastern Cape), Envir Karim (KZN) and Sereme Maduna (Free State). At the same congress, a motion to effect a major change to the structure of SASOP was adopted at the annual general meeting (AGM): that SASOP be registered as a Section 21 company (reg. no. 2007/012757/08), and the organisation has functioned in this capacity since May 2009 with the amendment and approval of its Memorandum and Articles of Association, during the AGM of the Society at the Arabella Hotel, Kleinmond. The SASOP Executive Committee was restructured as the SASOP Board of Directors, with the SESIG national convener as 1 of the 7 directors and voting members of the Board. The other directors on the Board are the SASOP president, past-president, president-elect, honorary secretary, honorary treasurer and the convener of SASOP Psychiatrists in Private Practice Special Interest Group ('P3' SIG).

It was undertaken by the convener and the new SESIG committee in 2009 to: communicate regularly with the SESIG constituency; establish contact with provincial roleplayers; liaise within SASOP, e.g. with colleagues in private practice; engage with managers and managerial representatives on provincial and facility level; and explore the discussion with academic institutions on joint appointees' positions regarding their dual academic and service provision responsibility. SESIG members were advised the time that, with SAMA as the only labour organisation for the medical profession, liaison may be essential to organise and position state- 
employed psychiatrists within the context of state-employed specialists in particular and medical practitioners in general. Several regional visits to subgroups were made during 2009 and 2010, including Eastern Cape, Western Cape, KZN, and Northern Gauteng. From 2009, regular analyses were made of the data on state employees included in the SASOP database.

The national SESIG committee for 2010 - 2012 included Robin Allen (Western Cape), Stephan van Wyk (Eastern Cape), Suvira Ramlall (KZN), Bernard Janse van Rensburg (Southern Gauteng), Kagisho Maaroganye (Northern Gauteng), Matshele Kewana (Limpopo), Marina Olivier (registrar) and Richard Nichol (Free State). A SESIG Strategic Statement for 2010 - 2012 noted that, firstly, as long as SASOP remains an affiliated organisation to SAMA, and as long as SAMA - through its 'borrowed' Democratic Nursing Organization of South Africa (DENOSA) seat in the public sector bargaining chamber - remains the only representative body for stateemployed medical practitioners on public sector remuneration matters, state-employed psychiatrists will have to continue to involve themselves as SESIG and SASOP members with SAMA and its structures much more concertedly, to ensure that state psychiatry and mental health issues in general are addressed. Alternatively, it was also in fact considered to either become part of the recently established private specialist body (the South African Private Practice Forum (SAPPF), which disaffiliated from SAMA in 2008) and to join these private specialists in their structure and endeavours, or to establish a parallel structure on the same basis as SAPPF, to provide another option of a more effective alternative 'labour union' type of organisation for state-employed specialists from different disciplines.

Consequently, the SESIG agenda and activities during the past 2 years included: SAMA liaison; liaison with the Rural Health Advocacy Project regarding mental health structures and services in Limpopo; the EDL and STG process; liaison with the national Department of Health; as well as the development of guidelines for standards in South African state sector psychiatric practice. These guidelines, in the format of a number of position statements on different issues, were developed through a national representative SESIG strategic workshop. This first strategic planning meeting was initiated by lan Westmore, SASOP President 2010 - 2012, and took place in Windhoek, Namibia, in March 2012. It was envisaged that this meeting would be the first of several similar regular annual strategic meetings in the future.

Ethics clearance was obtained during 2009 from the WITS Human Research Ethics Committee for a study entitled 'Profile of State Employee Members and Scope of the SESIG of the SASOP'. The purpose of the study was to review and document the policy, activity and progress of SESIG within its mandate as a specific subcommittee of SASOP. The objectives were to: describe the demographic and occupational profile of state employee members of SASOP; and identify strategic activities, set priorities

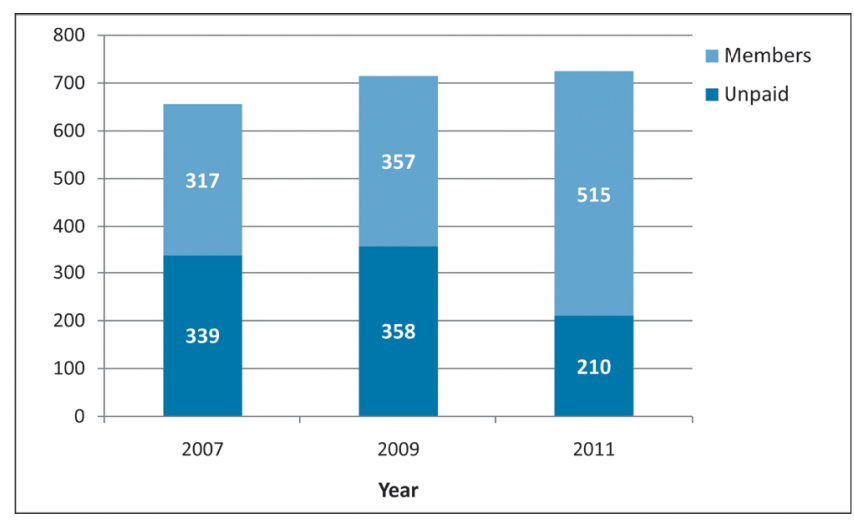

Fig. 1. Total number of psychiatrists registered as potential SASOP members.

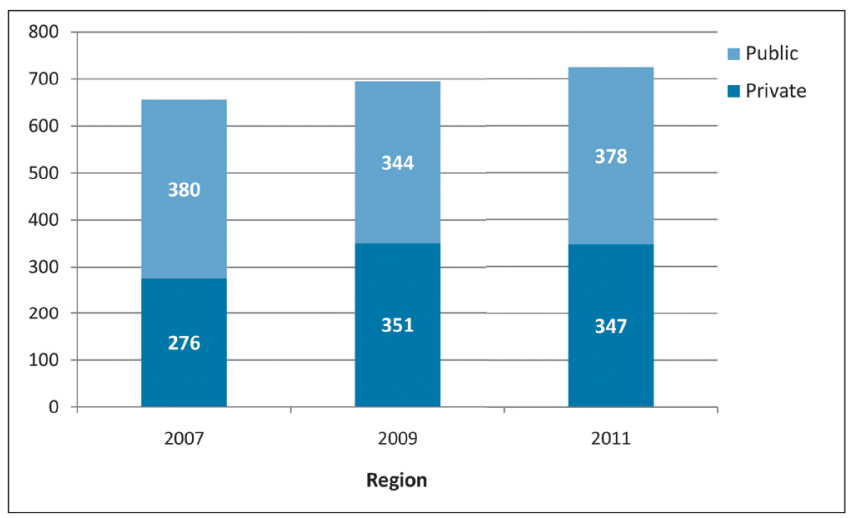

Fig. 2. Total number of state employees (psychiatrists and registrars) registered as potential SASOP members.

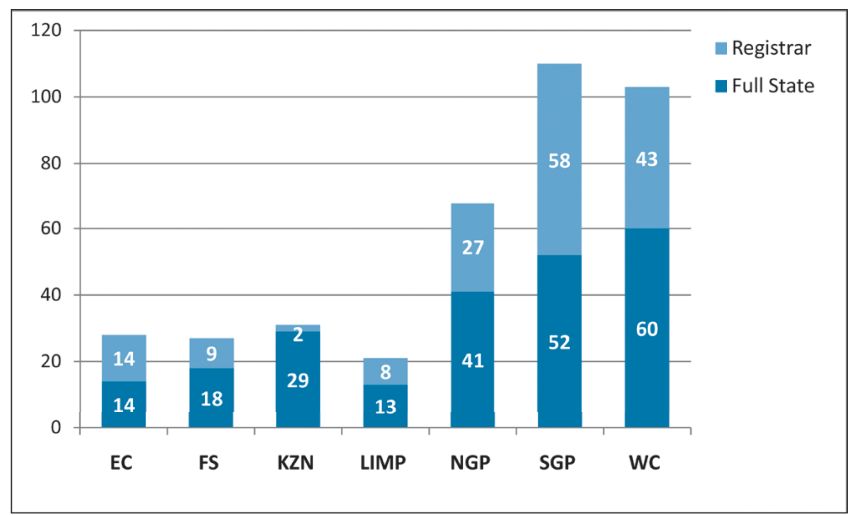

Fig. 3. Total number of psychiatric registrars registered as potential SASOP members per region. $E C=$ Eastern Cape; $F S=$ Free State; $K Z N=K w a Z u l u-N a t a l ; L I M P=$

Limpopo; NGP = Northern Gauteng; $S G P=$ Southern Gauteng; WC = Western Cape.

and establish measures for progress within the different areas of SESIG's mandate.

\section{Method}

This investigation included a retrospective quantitative review of the demographic and occupational profile of SESIG's members, as 
Table 1. Percentage of potential number of state-employed psychiatrists as paid-up SESIG members

\begin{tabular}{|c|c|c|c|}
\hline & 2007 & 2009 & 2011 \\
\hline Potential SESIG, $N$ & 380 & 344 & 378 \\
\hline Potential SASOP, $N$ & 656 & 715 & 725 \\
\hline Percentage potential SESIG of potential SASOP, $\%$ & 58 & 48 & 52 \\
\hline \multicolumn{4}{|l|}{ Paid-up SESIG, $n(\%)$} \\
\hline Eastern Cape & $9(6)$ & $13(8)$ & $16(8)$ \\
\hline Free State & $9(6)$ & $15(9)$ & $17(8)$ \\
\hline KwaZulu-Natal & $9(6)$ & $11(6)$ & $17(8)$ \\
\hline Limpopo & $11(8)$ & $5(3)$ & $11(5)$ \\
\hline Northern Gauteng & 27 (19) & $24(14)$ & $39(19)$ \\
\hline Southern Gauteng & $37(26)$ & $35(20)$ & $48(24)$ \\
\hline Western Cape & $41(29)$ & $33(20)$ & $54(27)$ \\
\hline Total & $144(100)$ & $174(100)$ & $202(100)$ \\
\hline Proportion of potential total SESIG members, \% $(n / N)$ & $38(144 / 380)$ & $50.6(174 / 344)$ & $53.4(202 / 378)$ \\
\hline Total SASOP paid-up members, $\%$ & 48.3 & 50 & 71 \\
\hline
\end{tabular}

captured by the SASOP database of current and potential members, as well as a review of the policies and processes by which strategic activities, priorities and measures for progress were identified within the different areas of SESIG's mandate.

\section{Results}

In 2007, 48.3\% (317) of the total potential number of psychiatrists were paid-up SASOP members. This proportion changed to $50 \%$ in 2009 (357) and to $71 \%$ (515) in 2011 (Fig. 1). Of this, there were $380(58 \%)$ total potential state-employed members in 2007, 344 (48\%) in 2009 and 378 (52\%) in 2011 (Fig. 2). In 2011, 40\% of the total membership was comprised of psychiatric registrars (Fig. 3). In 2007 , only $38 \%$ (144) of the potential total number of 380 of stateemployed psychiatrists were paid-up SESIG members. In 2009, this changed to $50.6 \%$ (174) and in 2011 to $53.4 \%$ (202) (Table 1). The Eastern Cape, Free State and Northern Gauteng subgroups had the biggest percentage of members per region in 2007, which changed in 2011 to Northern Gauteng, Western Cape and Eastern Cape.

\section{Discussion}

While several limitations must be considered about the accuracy of the SASOP database, especially in the updating of the total number of registrars per region, these figures on the potential and actual membership of SASOP and SESIG reflect a significant and consistent under-registration of psychiatrists and registrars as SASOP members over the past 5 years. This fact has been attributed to a pervasive lack of involvement of potential members, who often posed the question: 'What does SESIG/SASOP do for me?' Potential members seem to be doubtful about the benefits of being a SASOP and SESIG member and seem to want to be convinced that their interests are adequately protected. At the same time, it has been observed during the past 4 years, since SASOP changed its structure to that of a Section 21 company, and since SAMA had to adopt a new role as 'labour union'for the medical profession, that the very constituency who are doubtful about the usefulness of membership, is required to establish the mandate of the elected representatives during a particular term of office. SESIG as an organisation will therefore continue to be only as strong, relevant and representative as much as its potential membership participates in activities and becomes active in the different processes on regional and national levels.

Over the past 4 years, SESIG's profile relative to private practice colleagues, for instance, has been significantly improved by its convener being an equal voting member of the SASOP Board of Directors. SESIG still, however, currently faces important challenges concerning: the formulation of mental healthcare policy in the country, inter alia regarding implementation of the Mental Health Care Act, no. 17 of 2002; the public sector service rendering framework, and the expected implementation of a National Health Insurance (NHI) system, as well as the referral of patients between private and state sectors and vice versa; the co-ordination of the EDL and STG processes on regional and national levels (including the submission to decision-makers of adequate levels of evidence for the effectiveness of psychiatric drugs); identification of adequate standards; and the clarification of levels and definitions of services and facilities (e.g. primary, secondary and tertiary; and 72-hour assessment, acute units and psychiatric facilities). Challenges also still exist concerning: adequate funding and prioritisation; balancing and negotiating joint employees' added academic responsibility of training and research; establishing effective national and regional mental healthcare planning and monitoring processes in which psychiatrists have an active role; and ensuring appropriate national and provincial structures with effective communication between roleplayers. 
In the above matters, possibly the most important achievement during the past 2-year term of office has been the convening of the first annual SESIG strategic planning meeting in March 2012. This meeting was convened by the SASOP Board of Directors with the SESIG Committee, the various academic heads of department and representative clinical unit heads and other state-employed clinicians, including registrar representatives, from SASOP's different regional subgroup membership. The purpose of this strategic workshop meeting was to actively engage with a core of representative state sector SASOP members on the current most pertinent issues in state psychiatry and to develop a short- to medium-term strategy to effectively address these issues regionally and nationally. The outcome of these sessions with these members has informed SASOP's position on different issues and will be communicated formally as specific position statements or resolutions on the identified issues. One specific intended outcome of this meeting was also to inform SASOP's participation in the National Mental Health Summit held in April 2012. Presentations and discussion during the strategic SESIG meeting covered the following 6 areas: (i) the scope of state sector practice; (ii) pertinent policies for state practice; (iii) planning per region; (iv) teaching and research; ( $v$ ) accepted principles for care; and (vi) strategic mobilisation in view of set objectives.

The perspective of the discussions during this meeting was that 'while there is no health without mental health, there is also no complete mental health without psychiatry.'A number of key issues were identified, including: infrastructure and human resources; psychiatric EDL and STG; mental health and psychiatric disorders in the context of the global burden of disease (including HIV and substance-related problems); community-centred psychiatric services and referral levels; a recovery framework; culture, mental health and psychiatry; and psychiatry's specialty status and subspecialties. Twelve position statements were formulated to guide SASOP/SESIG activities during 2012 - 2014, including statements on: national mental health policy; psychiatry and mental health; infrastructure and human resources; STGs and EDL; HIV in children and adults; substance abuse and addiction; community psychiatry and referral levels; recovery and re-integration; culture, mental health and psychiatry; the specialty status of South African psychiatry; and forensic psychiatry.

Since the constituting of SESIG in 2000, SASOP has experienced a transition from a 'tea club' to a registered company in 2009. During 2009 and 2010, the disaffiliation from SAMA of private specialists to form the new SAPPF was noted. This organisation, representing several private management groups including the Psychiatric Management Group (PsychMG), successfully launched a legal challenge in 2010 against the national Department of Health about the appropriate publication of the Health Care Reference Price List (RPL). This has sparked suggestions that state-employed specialists should establish a similar dynamic and effective representative body to represent their interests in the public sector. State employees, however, are required by law to belong to a representative trade union. In addition to the inclusive National Health Workers Union (NEHAWU), SAMA still remains the only registered trade union alternative for the medical profession. For this reason, several meetings with SAMA officials and structures were attempted during 2010 and 2011. SAMA, through its Public Sector (PUBSEC) Committee and sub-structures (JUDASA, SEHDASA, SARA, ADASA) as well as its regional branches has, however, to a lesser or greater degree, been experienced during the past 2 years as generally inappropriately structured to adequately address the interests of specialists, and particularly those of state-employed psychiatrists.

\section{Conclusion}

The SASOP's SESIG also seems to be one of the only specialist associations with a separately structured special interest group which specifically focuses on state sector issues. With, however, only 50\% of the potential SESIG membership currently involved in the organisation and with a pervasive lack of involvement of senior state and joint employed academics, as well as of psychiatrists and registrars, the challenge remains of how a small specialist group such as SESIG with potentially only about 350 members in total, can effectively lobby and mobilise adequate support across specialist groups from different disciplines in the state sector. In the current trade union environment, SESIG may continue to be challenged to re-orientate towards improved representativeness, greater involvement from its constituency, to obtain mandates, to be accountable and to ensure appropriate regional and national structures.

While the SESIG convener and committee are currently elected representatives from a larger constituency, they are still operating on a 'voluntary' and unpaid basis. This may not be a sustainable position over the long term. However, by having established the first step of an ongoing strategic process to regularly re-assess progress and outcome, it is possible that further goals may be achieved to establish SESIG as a dynamic, effective and responsive group that will in fact be experienced on regional and national levels to be able to promote, maintain and protect the honour and interest of psychiatrists in the employ of the state, serving psychiatric patients within the public sector, as well as the interests of such patients.

\footnotetext{
References

1. Janse Van Rensburg ABR. A framework for current public mental health care practice in South Africa. Afr J Psychiatry 2007;10:205-209.

2. Rangaka T. South African Human Rights Commission Public Enquiry. African Journal of Psychiatry 2007;10:233-239.
} 